\title{
Monitoring the Dynamic Web to respond to Continuous Queries
}

\begin{abstract}
Continuous queries are queries for which responses given to users must be continuously updated, as the sources of interest get updated. Such queries occur, for instance, during on-line decision making, e.g., traffic flow control, weather monitoring, etc. The problem of keeping the responses current reduces to the problem of deciding how often to visit a source to determine if and how it has been modified so that a user response can be updated accordingly. On the surface, this seems to be similar to the crawling problem since crawlers attempt to keep indexes up-to-date as users pose search queries. We show that this is not the case, both due to the inherent differences between the nature of the two problems as well as the performance metric. We also develop and evaluate a multiphase solution to the problem. Some of the important phases are: The monitoring phase, in which changes, to an initially identified set of relevant pages, are tracked. From the observed change characteristics of these pages, a probabilistic model of their change behaviour is formulated and weights are assigned to pages to denote their importance for the current queries. During the next phase, the Resource Allocation phase, based on these statistics, resources, needed to continuously probe these pages for changes, are allocated. Given these resource allocations, the scheduling phase produces an optimal achievable schedule for the probings. An experimental evaluation of our approach compared to prior approaches for crawling dynamic web pages leads to some interesting observations pertaining to the differences between the two problem of crawling - to build an index - and the problem of change trackingto respond to continuous queries.
\end{abstract}

\section{INTRODUCTION}

The World Wide Web consists of an ever-increasing collection of decentralized web pages that are modified at unspecified times by their owners. Current search engines try to keep up with the dynamics of web by crawling it periodically, in the process building an index that allows better search for pages relevant to a topic or a set of keywords. Clearly, any good crawling technique needs to consider the change behaviour of web pages. Even in the best-case scenario, a crawler visits a web site only once in a few hours. This type and frequency of crawling is insufficient to handle a class of queries known as Continuous queries [12] in which the user expects to be continuously updated as and when new information of relevance to his/her query becomes available. For example, consider a user who wants to monitor a hurricane in progress with the view of knowing how his/her town will be affected by the hurricane. Obviously, a system which responds taking into account the

Copyright is held by the author/owner(s).

WWW2003, May 20-24, 2003, Budapest, Hungary.

ACM xxx. continuous updates to the relevant web pages will serve the users better than another which, say, treats the query as a discrete query, i.e., returns an answer only when the query is submitted.

Not surprisingly, the problem of keeping track of the dynamics of the web becomes inherently different for the continuous query case compared to classical(discrete) query case. For continuous queries, since the system should maintain the currency of responses to users, the problem translates to one of (a) knowing which pages are relevant, (b) monitoring the pages, to determine the characteristics of changes to these pages, and from these (c) deciding when to probe the pages for changes, so that responses are current. The last problem has several subproblems: allocating the resources needed for probing the pages, scheduling the actual probings, and then carrying out the probing. The work involved in handling continuous queries is portrayed in Figure 1. Here, the feedback arcs from the probing phase to the earlier phases indicate that observations made during the probing phase are used to tune subsequent decisions. We

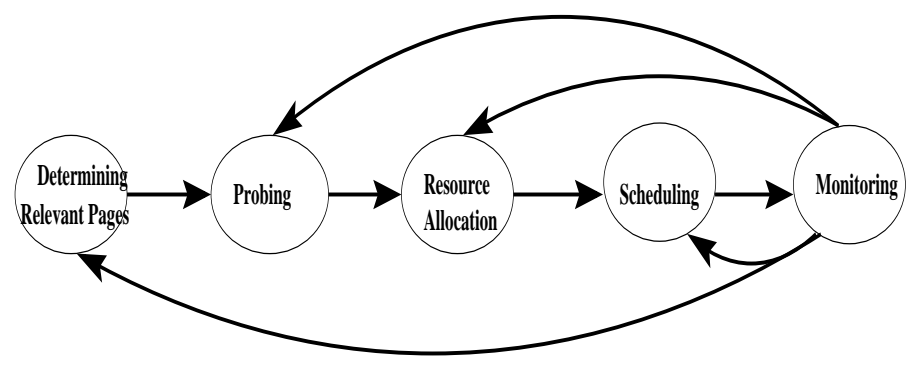

Figure 1: Different phases of our approach

use the term probings to explicitly account for the differences from the classical crawling problem. A probe fetches a web page, much like what a crawler does, but with the goal of fetching new information relevant to one or more queries while a crawl is not done with any specific user request in mind. Specifically, in this paper, our problem reduces to the distribution of a given number of probings among the pages whose changes need to be tracked to respond to a set of continuous queries.

It could be argued that discrete queries asked periodically with some time interval can be considered to be equivalent to continuous queries but the following reasons should help in dispelling this misconception: Firstly, determining the next time when the discrete query should be posed by the user is highly non-trivial. If the timeinterval is kept small then it may induce unnecessary load on the system, particularly when the updates are not frequent. If we set the time-interval to be large, it may lead to loss of information if more frequent updates, than expected, take place. Secondly, con- 
tinuous queries have a non-zero lifetime and so a query system can study a query's characteristics carefully and can answer it better than in the case where discrete queries, which have zero lifetime, are continuously posed. Furthermore, unlike in the case of discrete queries, the time taken to provide the system's first response to a continuous query may not be as important as the maintenance of currency during all the responses. To this end, our optimization metric is defined to minimize the information loss compared to an ideal probing algorithm which probes upon every change of a page.

So it should be clear by now that not only the nature of the crawling problem but optimization goals also become different when we move from discrete to continuous query case.

The rest of this paper is structured as follows: In Section 2, we define the problem formally and also provide an overview of the solution ???

\section{OVERVIEW OF OUR SOLUTION}

Consider a user who is worried about a hurricane in progress and wants to keep abreast of the hurricane-related updates. To achieve this, he poses a continuous $m$-keyword query $q=\left\{w_{1}, w_{2}, \ldots w_{m}\right\}$.

Identifying Pages relevant to a set of queries: Based on the keywords specified by a user, we need to first identify pages relevant to this query. The query is considered as a bag of terms and is fed to a classical search engine which in turn returns back a set of pages it finds relevant to queries using inverted index. We find, say, that the National Hurricane Center, National Weather Organization, and other tropical cyclone sites as well as news sites are relevant. The relevance of a page to a query can be measured by standard IR techniques based on the Vector-Space model: Given a $n$-word document $a=\left\{w_{1}, w_{2}, \ldots w_{n}\right\}$ and a set of $n$ recognized words, one can represent $q$ and $a$ each as a vector of word frequencies $\vec{q}$ and $\vec{a}$. A common measure of similarity between two word frequency vectors $\vec{a}$ and $\vec{q}$ weighted by inverse document frequency (idf) is the cosine distance between them:

$$
\operatorname{score}(\mathbf{q}, \mathbf{a})=\frac{\sum_{w \in q, a} \lambda_{w}^{2} \cdot f_{q}(w) \cdot f_{a}(w)}{\sqrt{\sum_{w \in q}\left(\lambda_{w} f_{q}(w)\right)^{2} \cdot \sum_{w \in a}\left(\lambda_{w} f_{a}(w)\right)^{2}}},
$$

where $f_{d}(w)$ is the number of times word $w$ appears in the document $d$ and $\lambda_{w}$ is the inverse document frequency of the word $w$ defined as:

$$
\lambda_{w}=\log \left(\frac{|\mathcal{D}|}{\left|\left\{d \in \mathcal{D}: f_{d}(w)>0\right\}\right|}\right)
$$

where $\mathcal{D}$ is the document set in consideration.

Monitoring the Relevant Pages to Characterize Changes: Once relevant pages have been identified, by visiting each page at frequent intervals during a monitoring period, changes to these pages are monitored, update statistics collected, and the relevance of the changes, vis a vis the queries, is assessed. This is used to build a statistical model of the changes to the pages relevant to a set of queries. These statistics include page update instances, page change frequency, and relevance of pages for queries.

With this information in hand, we can move to the next set of phases where resource allocation and scheduling decisions are made concerning the probings to be done for an interval of length $T$. The basic idea is that these scheduling intervals of length $T$ repeat every $T$ units of time and we will make decisions pertaining to the probings to be carried out in one scheduling interval using both new data and the results from the previous scheduling intervals.

In general, based on the results of probings carried out for $T$ time units, scheduling, resource allocations, change statistic computations, and page relevance can all be revisited. These, as mentioned earlier, correspond to the arcs going from the "probings" phase to the earlier phases of Figure 1.

Let $C$ denote the total number of probings that can be employed in a single scheduling interval. $C$ is derived as an aggregation of the resources needed for probing, including CPU cycles, communication bandwidth, and memory. ${ }^{1}$ With this information in hand, decisions are made about the allocation of a given number of probings among a set of pages while also deciding when these allocated probings should ideally occur.

Resource allocation phase: This phase decides the time instances at which these allocated probings should be done. In scheduling phase, we take these time instances as inputs and prepare a feasible schedule to meet our optimization measures. The overall goal of these two phases is to probe in such a way that the probes occur just after updates are expected to take place. The number of lost updates is an indication of the amount of lost information and minimizing this is the goal of the system.

We now give a summary of the resource allocation and scheduling phases using the following model (The basic general model is adopted from [16] and is modified to suit our problem definition.) We denote by $\mathrm{N}$ the total number of web pages that need to be monitored, which shall be indexed by $i$. Let $P$ denote the set of web pages relevant to the continuous queries. $\lambda_{i}$ is the estimated number of changes that occur in page $i$ in $T$ time units. Henceforth we will refer it as change frequency for page $i$. page may get updated.

Suppose $U_{i}$ denotes the sequence of time instances $u_{i, 1}, u_{i, 2} \ldots \ldots . u_{i, p_{i}}$ at which possible updates occur in page $i$. Here, $p_{i}$ is the total number of update instances for $i^{t h}$ page, i.e., cardinality of sequence $U_{i}$ $\left(P_{i}=\left|U_{i}\right|\right)$. Note that it is not certain that a page would be updated at these time instances and so there is a probability $\rho_{i, j}$ associated with each time instance $u_{i, j}$ that denotes the chances of getting this $i^{t h}$ page updated at the $j^{\text {th }}$ instance.

We assume $0 \leq u_{i, 1} \leq u_{i, 2} \ldots . . u_{i, p_{i}} \leq T$ and $u_{i, 0}=0$ and $u_{i, p_{i}}=T$. It should be clear that if we decide to probe at some instance, then it should be at the potential update time instance only because there is no reason to delay it beyond when a update might occur. If number of probings allocated for a page is equal to the number of update instances, then we can always maintain a fresh copy of this page by probing at all possible update instances. But in practice we will not be able to perform as many probings as the number of update instances. So we need to pick a set of update instances at which only this page would be probed and not at others. Hence with every update time instance, we associate a variable $y_{i, j}$ where

$y_{i, j}=1$ if probing of $i^{\text {th }}$ is done at time $u_{i, j} 0$, otherwise

if we probe the $i^{\text {th }}$ page $x_{i}$ times, then $\sum_{j=0}^{p_{i}}\left(y_{i, j}\right)=x_{i}$ holds.

Scheduling the probes: This involves taking the ideal timings for the probings of each page and obtaining an optimal achievable schedule out of it. We map this problem to flow-shop scheduling problem [13] with the goal of minimizing the average completion time. Though this problem has been proved to be NP-Complete [11], there are fast approximation algorithms [2] that provide an approximate bounded solution. Next we probe these pages according to the designed schedule and when this scheduling interval is finished, we go back to query system and update the characteristics

${ }^{1}$ For example, the authors of [9] report that with two $533 \mathrm{MHz} \mathrm{Al}-$ pha processors, 2 GB of RAM, 118 GB of local disk, and a 100 $\mathrm{Mbit} / \mathrm{sec}$ FDDI connection to the Internet, Mercator under srcjava, their crawler crawled at an average download rate of 112 documents/sec and $1,682 \mathrm{~KB} / \mathrm{sec}$. Similarly the capabilities of a given infrastructure can be mapped to the number of probings that it is capable of on average. 
of these pages on the basis of the observations done in the preceding scheduling interval.

\section{OPTIMAL RESOURCE ALLOCATION}

As noted earlier, we need to distinguish between pages on the basis of two metrics. One is the nature of page-change behaviour and the other is importance of page for queries. Page-change behaviour is studied during a monitoring phase and is denoted by associating a probability of change with every update instance. Next we would show the way in which pages can be ranked by assigning weights to them using relevance measures. These relevance measures are determined for each page for each query during monitoring period.

\subsection{Importance of Specific Pages}

\section{Glossary}

$Q:$ Set of all queries submitted in the system.

$Q_{p_{i}}$ : Set of queries for which $i^{\text {th }}$ page is found to be relevant in monitoring period.

$w_{i, j}$ : Estimated relevance of $i^{t h}$ page for $j^{\text {th }}$ query. It would be positive for all queries q $\varepsilon Q_{p_{i}}$ and zero for all $q \varepsilon Q Q_{p_{i}}$. It would be computed during monitoring period.

$\omega_{i}$ : Importance of $i^{t h}$ query. This would be an input to the system. $W_{i}$ : Weight of $i^{t h}$ page. It is computed as shown below.

It is clear that not all pages would be equally important for each query in the system. So we would rank pages by assigning weight to each page using its relevance measures for queries. The weight of a page denotes the value of current copy of page and if page gets updated before the current copy is probed, we assume that it incurs us a loss of $W_{i}$ value. Suppose there are $Q$ queries submitted in the system. Each page would be relevant for a set of queries. Say the $i^{\text {th }}$ page is relevant for a query set denoted by $Q_{p_{i}}$. Also the sequence of values measuring relevance of $i^{t h}$ page for each query is $w_{i, 1}, w_{i, 2} \ldots \ldots w_{i,\left|Q_{p_{i}}\right|}$. Note that these relevance measures would be calculated during monitoring period and after every $T$ time units when a scheduling interval is finished, it would be updated based on the earlier values and values found in last scheduling interval.

Also let there be Importance measure associated with each query because of the classification of users submitting the query or classification of query domains, say $\omega_{1}, \omega_{2} \ldots . . \omega_{Q}$. So now we can measure the weight $W_{i}$ of $i$ th page as

$$
W_{i}=\sum_{j \varepsilon Q}\left(\omega_{j} w_{i, j}\right)
$$

where $\mathrm{Q}$ is a set of queries in the system, $\omega_{j}$ is importance of $j$ th query,

$w_{i, j}$ is relevance measure of $i$ th page for $j$ th query and is greater than 0 only if $q_{j} \varepsilon Q_{p_{i}}$

\subsection{Goals of the Resource Allocation Phase}

For Continuous queries, our aim is to minimize weighted importance of changes that are not reported to users. It could be formulated as

$$
\min \sum_{i \varepsilon P}\left(W_{i} E_{i}\right)
$$

where $E_{i}$ denotes expected number of lost changes for $i$ th page

As we have assumed that each update instance is independent of others,

$$
\text { so } E_{i}=\sum_{j \varepsilon U_{i}} \rho_{i, j}\left(1-y_{i, j}\right)
$$

Resource constraint is given by

$$
\sum_{i \varepsilon P} \sum_{j \varepsilon U_{i}} y_{i, j}=C
$$

Note that here we are assuming that in $T$ duration, relevance of each updated copy of page for queries remains the same as estimated during monitoring period. After a scheduling interval is finished,we will update these relevance measures on the basis of relevance measures actually found in the interval preceding it. So unless $T$ is kept very large or page updates are very erratic, our assumption remains very practical. Also we are assuming that with each update of page, information of update preceding it is completely lost. That is, the number of lost updates is an indication of the amount of lost information. While this may not always be true, it gives us a simple way to state the goal to be accomplished.

In certain cases, it is possible that we would be having more information than the case described above. For example, if we can measure change behaviour of $i^{\text {th }}$ page with respect to $j^{\text {th }}$ query, then it would be possible to allocate resources even more efficiently. For example, suppose we get to know during monitoring period that a particular news site mainly declares health updates only once at the start of day and in rest of the time, it remains mainly concerned about political and sports updates, then we can characterize change behaviour of this page with respect to queries concerned with sports, medical and political domain.

Suppose $\rho_{i, j, k}$ denotes the probability of change of $i^{t h}$ page at $j^{t h}$ update instance where this change is relevant for query $k$. Then our resource allocation problem can be formulated as

$\min \sum_{i \varepsilon P} E_{i}$

where $E_{i}$ denotes the weighted expected number of lost changes for $i$ th page

$$
\text { So } E_{i}=\sum_{k \varepsilon Q} \omega_{k} \cdot w_{i, k} \cdot \sum_{j \varepsilon U_{i}} \rho_{i, j, k}\left(1-y_{i, j}\right)
$$

with resource constraint as

$$
\sum_{i \varepsilon P} \sum_{j \varepsilon U_{i}} y_{i, j}=C
$$

If we can extract even more information by measuring not only probability of change of $i^{\text {th }}$ page at update instance $u_{i, j}$ but also average importance of change at this time instance, then it would make resource allocation even more efficient. For example, suppose we found that a particular research site compiles and announces all its previous day's research updates daily at 10:00 a.m. in the morning and in rest of day, it updates its page only when some new research breakthrough takes place. Then it is clear that visit to this page at 10:00 a.m. is certainly more fruitful than any other visit to this page. It is easy to accommodate aforementioned extension in the above model, and so we won't concern ourselves with it due to lack of space. Is this last paragraph ok as it seems quite impractical. We may argue that though above extension seems to assume very much information, it may possible in continuous query case to actually have this much information because we do get a lot of time to observe pages in continuous query case.

\subsection{The resource Allocation Algorithm}

Both of the above formulated resources allocation problem are discrete, separable and convex.

1. Discrete: because variable $y_{i, j}$ can take only discrete values. Our problem is inherently discrete due to discrete nature of 
probings. Either a probing would be allocated to a page or it won't be. There can't be anything between these.

2. Separable: because optimizing function could be expressed in terms of $y_{i, j}$ only.

3. Convex: due to convex nature of optimizing function.

Discrete, Separable and Convex problems have been well-studied in theoretical Computer Science [10]. Formally it can be stated as shown below:

$\min \sum_{i=1}^{N} F_{i}\left(x_{i}\right)$

with resource constraint

$\sum_{i=1}^{N} x_{i}=R$

where $x_{i}^{\prime} s$ are discrete and $F_{i}^{\prime} s$ are convex. A greedy algorithm exists for the discrete case [7]. There is a faster algorithm also for our problem, due to Galil and Megiddo, which has complexity $O\left(N(\log R)^{2}\right)$. The fastest algorithm is due to Frederickson and Johnson [8] and it has complexity

$O(\max \{N, N \log (R / N)\})$. In our case, the output of these algorithms is a set of $y_{i, j}$ 's which get a value of 1 to meet our optimization measure. This set in turn gives us the number of probings allocated to a page $\left(x_{i}=\sum_{j=0}^{p_{i}}\left(y_{i, j}\right)\right)$ as well as the ideal time instances at which these allocated probings should be done.

\section{SCHEDULING OF PROBINGS}

At this point we know the number of probings allocated to each page and also the ideal time instances at which these probings are supposed to be done. In practice, we have a set of $M$ parallel processes which continuously perform these probings. Now our task is to schedule the probings among $M$ parallel probes. While determining any schedule, our aim is to minimize the total delay occurring between the ideal time instances and the actual scheduled time instances.

Let page $P_{i}$ be allocated $x_{i}$ number of probes in an optimal resource allocation. Also the time instances at which these $x_{i}$ probes should be employed are $t_{1}, t_{2}, t_{3} \ldots \ldots . . t_{x_{i}}$. Let $f e t c h_{i}$ be the average fetching time for $i^{\text {th }}$ page. The scheduling problem can be easily mapped to parallel shop scheduling problem.

In this problem, each job has to be processed on exactly one of $M$ identical machines. Each probing could be regarded as a job whereas the probing processes are equivalent to machines. Suppose there are a total of $n$ such jobs. In scheduling problems, the time at which a job becomes available for processing is called the release time $\left(r_{j}\right)$ and the time for which it needs to be run on a machine is called the processing time. So in our case, ideal probing time instances $t_{1}, t_{2}, t_{3} \ldots \ldots . . . t_{x_{i}}$ would be the release times and fetching times of pages would act as processing times $\left(p_{j}\right)$ for jobs. Our goal is to minimize the delay $d_{i}$ between ideal probing time instance $\left(r_{i}\right)$ and actual time instance $s_{i}$ of scheduling.

In our case all jobs are equally important as there is no weight assigned with each probing. So our problem could be formulated in scheduling notation as $R|M| r_{j} \geq 0 \mid \sum_{j} C m_{j}$ meaning that $R$ jobs of non-trivial release times are available for scheduling at $M$ machines with goal as minimization of average completion time. Here $\mathrm{Cm}_{j}$ denotes completion time for job $j$. It might not be clear how minimizing average completion time would lead to minimization of average delay time. It is because of the following equality :

Total Completion Time

$$
\begin{aligned}
& =\sum_{i=1}^{R}\left(C m_{i}\right) \\
& =\sum_{i=1}^{R}\left(s_{i}+p_{i}\right) \\
& =\sum_{i=1}^{R}\left(r_{i}+d_{i}+p_{i}\right) \\
& =\sum_{i=1}^{R}\left(d_{i}\right)+\sum_{i=1}^{R}\left(r_{i}\right)+\sum_{i=1}^{R}\left(p_{i}\right)
\end{aligned}
$$

As $\sum_{i=1}^{R}\left(r_{i}\right)$ and $\sum_{i=1}^{R}\left(p_{i}\right)$ are constants, minimizing average completion time is same as minimizing delay time. Note that $C m_{i}$ is the same as $s_{i}+p_{i}$ because of non-preemptive scheduling. Unfortunately even simpler problem $R|1| r_{j} \geq 0 \mid \sum_{j} C m_{j}$ don't have any polynomial time algorithm and has been proved to be NP-Complete [11]. So we have to look for approximation algorithms. As our problem is offline, there is 1.58-approximation algorithm [11] that could be used for it. We have used this algorithm in conducting our experiments.

\section{EXPERIMENTAL EVALUATION}

In this section, after explaining the setup for the experiments, we describe the results.

\subsection{Experimental setup and Performance Met- ric}

Comparison with Alternative Algorithms:

In previous sections, we proposed an optimal resources allocation policy for probing changes in pages relevant to continuous queries. Here we evaluate our policy by comparing it with some classical policies using synthetic a data set. These policies are Uniform and Proportional policy [4] in which resources(probings) are allocated uniformly across all pages or proportional to changefrequencies of pages respectively. As suggested in [16], it would be fair to compare with the weighted version of these policies than the unweighted ones: In the Weighted Uniform scheme, the number of probings $\left(x_{i}\right)$ allocated to a page depends on the weights $\left(W_{i}\right)$ associated with the page but is independent of its change frequency $\left(\lambda_{i}\right)$ : $x_{i} \propto W_{i}$. In the proportional scheme, $x_{i} \propto\left(\lambda_{i} * W_{i}\right)$.

Parameters of the Experiment: As mentioned earlier, each page has an estimated change frequency $\left(\lambda_{i}\right)$ associated with it which denotes the expected number of changes that occur in a page in $T$ time duration. Also there is a sequence of update instances $\left(u_{i, j}\right)$ for each page $\left(U_{i}\right)$ which enumerates the time instances at which changes can occur in a page. With each update instance $\left(u_{i, j}\right)$, there is an associated probability $\left(\rho_{i, j}\right)$ which denotes the probability with which a change can occur at this instance. In our experiments, to make it simple, we make this sequence of update instances $(U)$ the same for each page. Other parameters are decided as below :

1. $N_{q}$ : number of queries submitted in the system. It is set to 500 .

2. $N$ : number of pages found relevant for queries submitted. It is also set to 500 .

3. $C$ : number of probings available. It is varied from 1000 to 50000 in our experiments.

4. Change frequency distribution: The change frequencies $\left(\lambda_{i}\right.$ 's $)$ are chosen according to Zipf distribution with parameters $N$ and $\theta$. $\theta$ varies from 0 to 2 . Such distributions run the spectrum from highly skewed (when $\theta=2$ ) to uniform (when $\theta=0$ ). This distribution is henceforth referred as change frequency distribution. Unless otherwise specified, $\theta$ is set to 2 in experiments.

5. Update probability distribution : As said above, we have used a universal sequence of update instances $(U)$ in our experiments. In this universal sequence, update instances are uniformly distributed throughout the duration $T$ every $\delta$ time units. In our experiments, we have divided $T$ in 480 update instances. Probabilities $\left(\rho_{i, j}\right)$ associated with these update instances $\left(u_{i, j}\right)$ are varied between 0 and 0.3 and follow a 
Zipf distribution. Henceforth we will refer to this distribution as update probability distribution. Zipf is chosen because of the fact that most of the web pages have time durations when they are updated with greater probabilities in comparison to the rest of the time durations. News sites can have multiple hot time durations and that can be modeled by generating many "humps" in their update probability distribution with probability varying in the vicinity of every hump in Zipf fashion. Note that the probabilities $\left(\rho_{i, j}\right)$ for all update instances of a page should sum up to expected change-frequency $\left(\lambda_{i}\right)$ of that page. Also note that we vary the zipf parameter of this update probability distribution from 0 to 2 in our experiments and so we get a corresponding update probability distribution for a page in $T$ varying from a uniform to a highly skewed distribution. This makes our experiments free from any a priori assumptions about page change behaviour and helps in evaluating our policies for all possible scenarios.

6. Weight of queries : All queries are assigned the same importance measure $\left(\omega_{i}\right)$. It means that there is no distinction made among queries and they are defined to have equal importance.

7. Page Weight Distribution : Recent studies [15] show that popularity of pages vary in zipf fashion as shown in Fig 2 . Drawing an analogy, we make relevance of page $j$ for a query

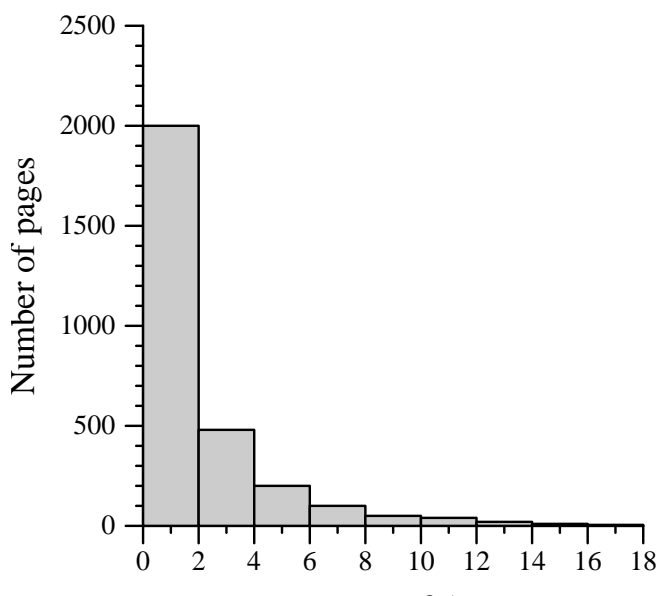

Frequency of Access

Figure 2: Observed Popularity Distribution

$i\left(w_{i, j}\right)$ to be distributed according to zipf distribution in our experiments. This distribution will be referred as query relevance distribution. Also the more dynamic a page, the more more popular it is too, as shown in [17]. So we make the important pages be more dynamic in our experiments by assigning maximum value of page relevance distribution to a page in a biased random manner. The summation of relevance measures of a page for all the queries gives us the weight $\left(W_{i}\right)$ for this page as shown in section 3 . The distribution according to which $W_{i}$ varies is referred to as page weight distribution.

8. probing ratio: denotes the ratio of the total number of probings to, $\left(\sum_{i \varepsilon P} \lambda_{i}\right)$, i.e., the number of actual changes expected in time $T$ time.0

Performance Metric: Returned Information ratio: Part of the information that is returned by a policy in a fixed number of probes is called as Returned Information Ratio. Using section 3, it turns out to be

$\frac{\sum_{i \varepsilon P} W_{i} * \sum_{j \varepsilon U}\left(\rho_{i, j} * y_{i, j}\right)}{\sum_{i \varepsilon P}\left(W_{i} * \lambda_{i}\right)}$

where $y_{i, j}$ is defined in the same way as in section 3 . Note that the maximum possible value of returned information ratio is 1 and it is attained when all those $y_{i, j}^{\prime} s$ are made 1 for which corresponding $\rho_{i, j}^{\prime} s$ are non-zero. This is the performance metric on the basis of which we compare various allocation policies in our experiments.

\subsection{Comparison of resource allocation policies}

In this experiment, we compare three aforementioned resource allocation polices and also observe the effects of update probability distribution and page weight distribution on their performance.

\subsubsection{When both page weights and update probabil- ities are uniformly distributed}

We make both these distributions uniform and set Zipf parameter of change frequency distribution to 2 as shown in Fig. 3 .

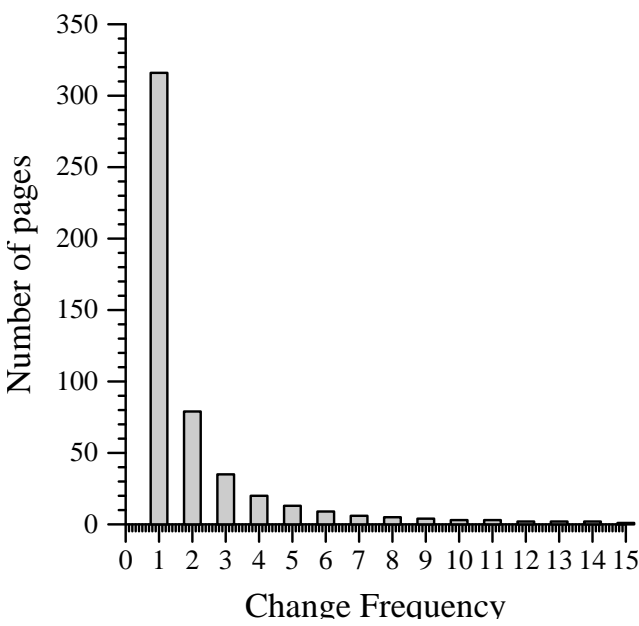

Figure 3: Change frequency distribution

Uniform page weight distribution means that all pages are having equal importance while uniform update probability distribution leads to equal probability of change to a page at any update instance in $T$. Fig. 4 shows the performance of different resource allocation policies. There are 2 important observations.

1. Proportional policy performs better than its Uniform counterpart. This is very surprising as all earlier studies showed the reverse to be true [4] [16]. The reason becomes pretty clear when we look into the nature of the crawling vs. the probing problem. In our case, we are answering continuous queries and naturally our aim is to detect as many changes as possible. So when all other parameters are uniform(pageweight and update probability distribution), one would certainly expect more benefit by probing those pages which are having high change frequency $\left(\lambda_{i}\right)$ because ultimately only these pages are having maximum chances of changing. This is what Proportional policy does and so it performs better than Uniform policy. Earlier studies solved the problem for answering discrete queries and aimed to maximize freshness 


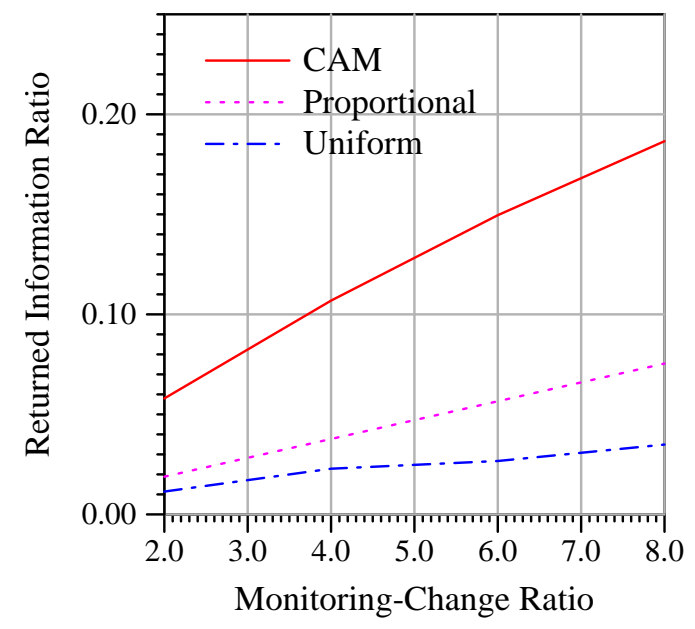

Figure 4: Under uniform weight and update probability distribution

of page which is found to be of convex nature. So the performance of Uniform becomes better than Proportional in their case. We offer a formal proof of why Uniform does not work as well as proportional at the end of the paper.

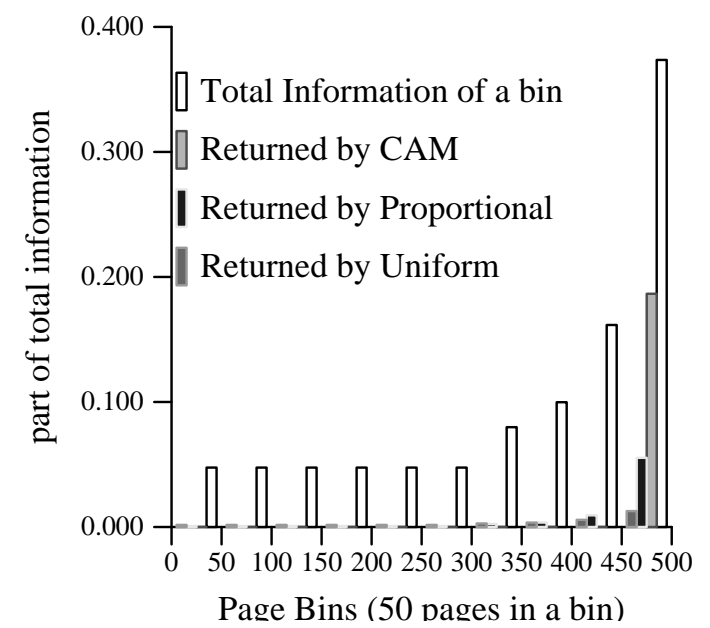

Figure 5: Characteristics of resource allocation policies

2. Optimal policy also allocates more probings to more dynamic pages but it does it even more aggressively than Proportional. Fig. 5 shows that Optimal allocates all its probings to only a few pages (for clarity, for the sake of this graph 50 pages of consecutive page indices have been grouped into a bin) and delivers most of the information to queries from these pages. Again the pages probed are those which have high probabilities of actually showing changes. Proportional too does this but it allocates probings in proportional manner only while Optimal does it in more biased way and so it gets even better performance. As it is evident from the graph that optimal policy performs $300 \%$ better than proportional policy and around $600 \%$ better than uniform policy!
Obviously now if we start decreasing the skewness, i.e., the zipf parameter, of change frequency distribution too, then policies start coming closer and in the extreme case, they all become the same when frequencies are made to be distributed in a completely uniform manner (Zipf parameter $=0)$.

\subsubsection{When page update probabilities are skewed}

Here, we skew the update probability distribution with zipf parameter being set to 1 . So pages are still of equal importance but for each page, the update instances are no more equi-probable, in experiencing a change. Fig. 6 shows the performance graph. for this data set. Again, optimal performs best leaving other allocation policies far behind. It is 12 times better than uniform policy. But in this case, the pages which are probed under optimal allocation turn out to be quite diversified as pages with even lesser change frequency have some update instances with a good chance of actually changing as shown in Fig. 6.

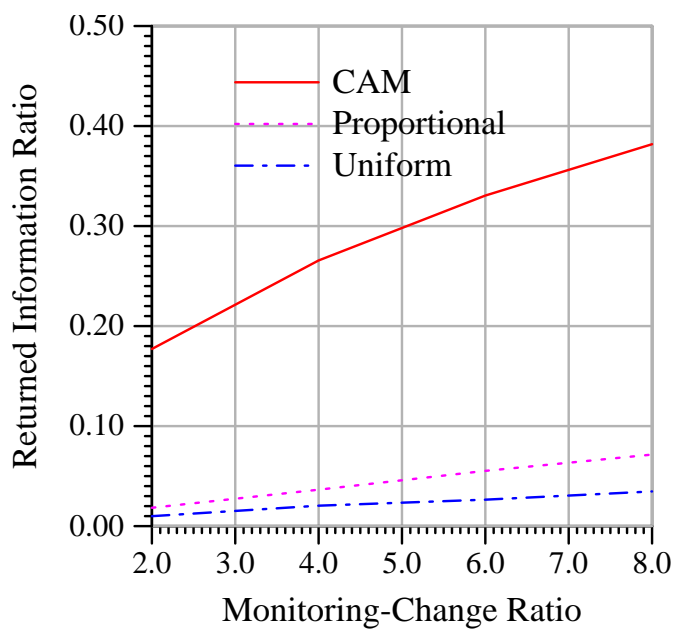

Figure 6: Under skewed update probability and uniform page weight distribution

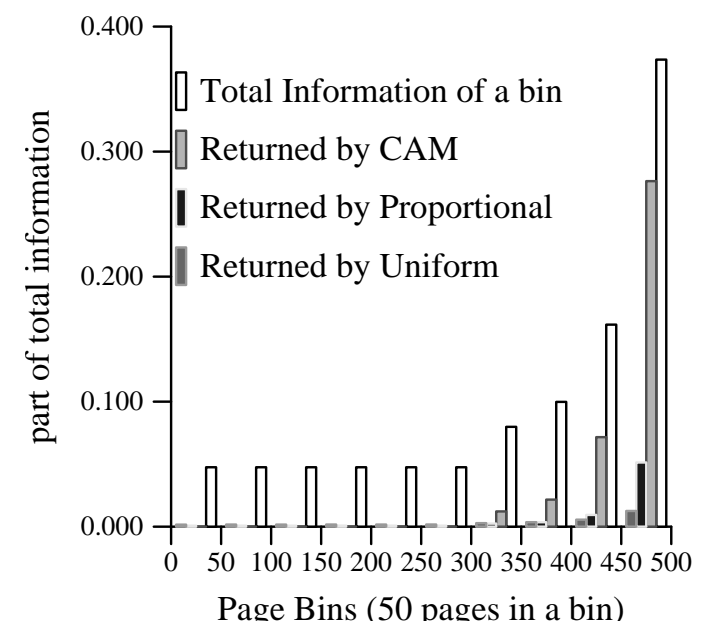

Figure 7: Characteristics of resource allocation policies 


\subsubsection{When page weights are skewed}

If we make page-weight distribution skewed while keeping update probability distribution uniform, we find that optimal again performs far better than others as shown in Fig. 8. Also, now it allocates probings to those pages which have high importance and a higher probability of getting changed.

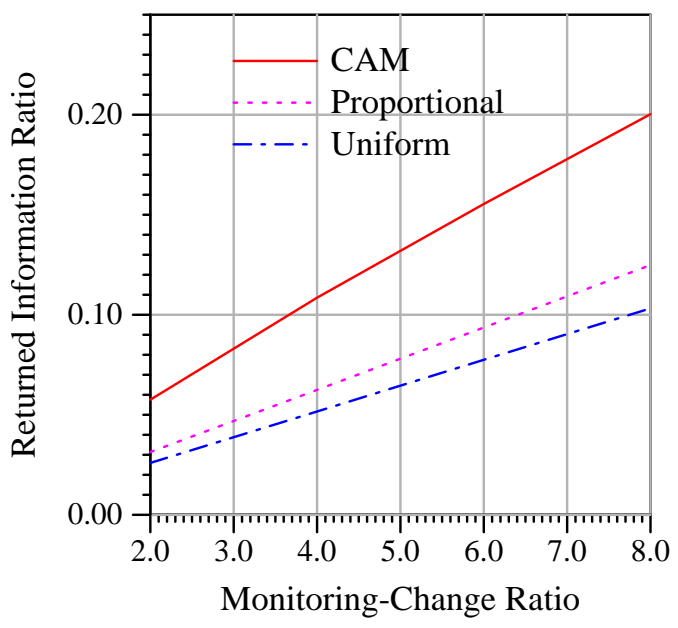

Figure 8: Under skewed page weight and uniform update probability distribution

\subsection{Effect of varying the skewness of the up- date probability distribution}

In this experiment, we compare our resource allocation policy with proportional and uniform policies under varying skewness of update probability distribution. This will also help us investigate the drawbacks of these classical approaches in probing web pages for answering continuous queries.

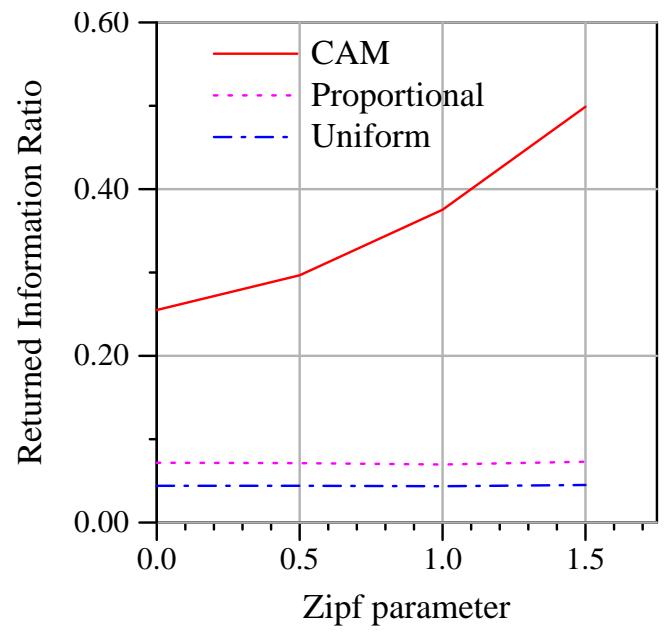

Figure 9: Under varying skewness of update probability distribution

Fig. 9 compares performance of different resource allocation policies when probing ratio is kept at 9 and page weight distri- bution is uniform. It is clear from the curves that for this data set, optimal resource allocation policy always performs better than other resource allocation policies. To emphasize the difference, we varied the update probability distribution keeping other parameters same as before. As evident from Fig. 9 that optimal resource allocation policy starts performing even much better than other policies as update distribution is made more and more skewed. It exhibits a 5-fold improvement over uniform resource allocation policy at zero zipf parameter but when zipf parameter is set to 1.5 , its performance sees a 10 -fold improvement.

This is because of the fact that in optimal resource allocation policy, a probing is made at those update instances which have high probability of returning relevant information and so as update probability distribution is made more and more skewed, it copes up with the skewness of data by selecting the most beneficial instances for probing and performs even better than before. But this is not the case with uniform and proportional policies as they do not look at the granularity level of update instances and decide probings only based on weight and change frequency. Note that when zipf parameter is set to zero, it does not mean that update probabilities become uniformly distributed, instead of this it means that all update probability values occur equal number of times.

\subsection{Identifying Parameters that produce Bet- ter Results for Continuous Queries}

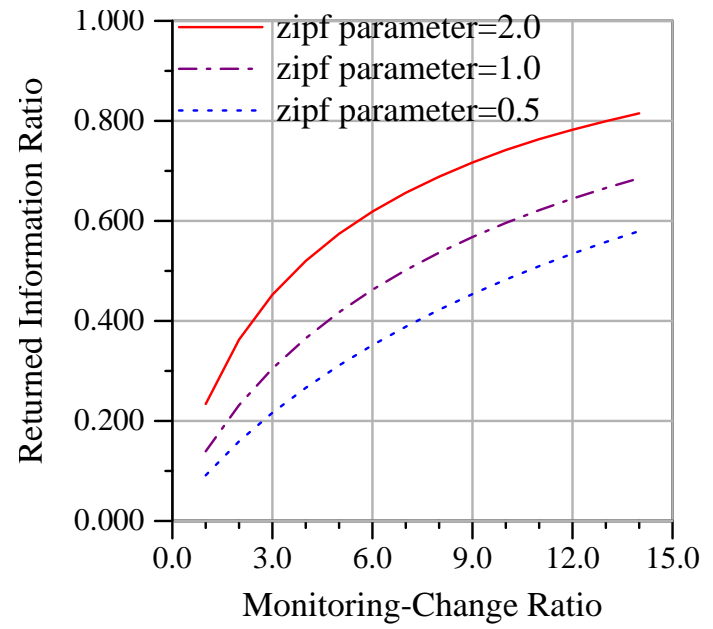

Figure 10: With varying skewness of update probability distribution

In the previous experiment, we observed that even when we have 9 times more probings available than expected number of changes in $T$, the loss of information remains significant. We feel that this is because of the distributed and uncertain nature of page change behaviour which make number of probings required for good performance very large(section 5.2.1). In the ideal case, we will require continuous monitoring of web pages and so even a large number of probings (until they become comparable to number of update instances) won't be of much help.

Fig. 10 shows how performance varies with the update probability distribution of page change behaviour. It is also evident that pages with almost uniform update probability distribution would affect the performance for continuous queries. We find that page weight distribution also affects the performance in a significant 


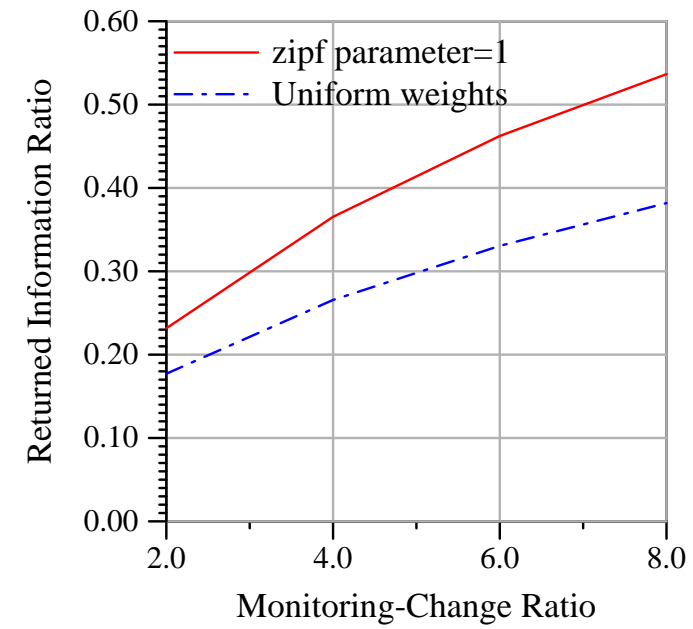

Figure 11: With varying skewness of page weight distribution

way. It is justified too because if we can somehow figure out during monitoring phase that a particular set of pages is serving a major part of reportings to users for answering query, then we can improve our performance by assigning them a major share of probings also. Fig. 11 shows the effect of page-weight distribution on the performance of allocation techniques.

Continuous queries can be responded to even more efficiently by extracting meta-information about the change behaviour of web pages. We need to have more knowledge of page characteristics to get a good performance for answering continuous queries .

\subsection{Effect of Probing Ratio on Continuous Queries}

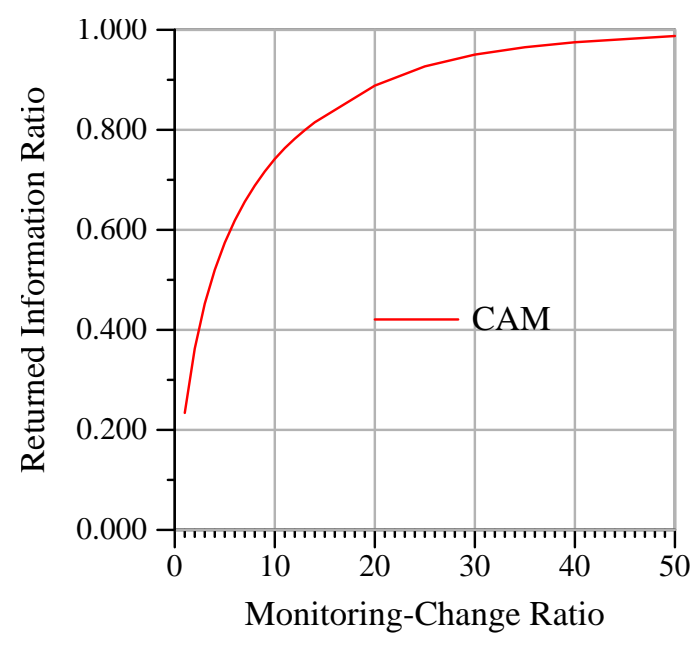

Figure 12: Performance curve for optimal policy

Here we evaluate the practical application of our proposed scheme. As evident from Fig. 12, 90\% of the Information is returned in our technique when probing ratio is 20 . Without using our probing technique, retrieving $90 \%$ of the information would require probing of at least 420 instances while our scheme reduces this to only 20 probings (5\% of blind probings). So it is indeed helping us in monitoring the pages but it is the uncertain nature of page changes which is actually impairing the performance.

\subsection{Reallocating resources}

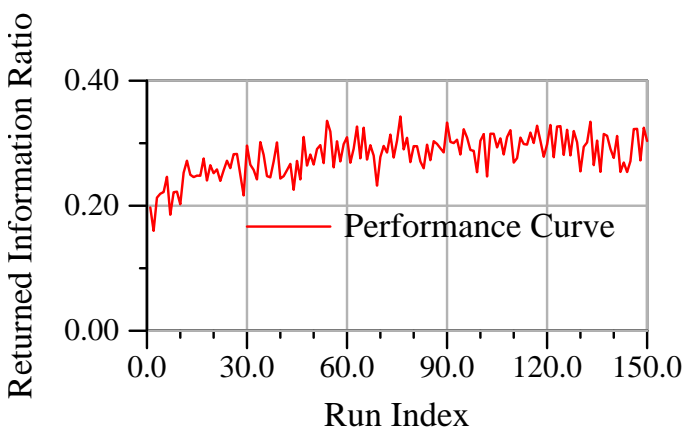

Figure 13: Effect of Resource Reallocation after every run

As we said while describing our technique that after every run of length $T$, we update page change behaviour and accordingly modify resource allocation for next run. But this may become very expensive especially when $T$ is small. We next study the effect of the the resource allocation delay.

We making many probing runs of period $T$ assuming that a brief monitoring period preceded it in which page change was fully captured and then we would show how performance varies with each run and with varying delays in repeating resource allocation. We start with update probability distribution of zipf parameter being set to 1 . Then we generate an actual event based on this update probability distribution by tossing a biased coin at every update instance and declaring a change at that instance if it falls head.

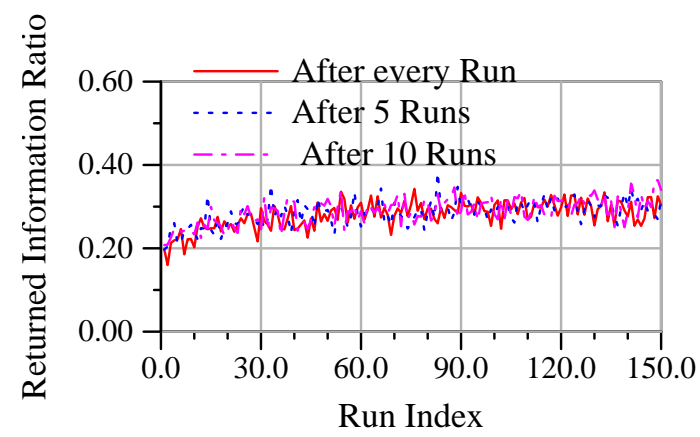

Figure 14: Effect of Varying the Resource Reallocation Delay

Before next run, we modify the update probability distribution based on this last run made by modifying update probabilities $\left(\rho_{i, j}\right)$ of those update instances which get probed in this run. We do this modification in very naive fashion by estimating average rate of occurring of updates at this update instance. So if a page got updated on 5 occasions in last 10 probings to this page at a certain update instance, we assign 0.5 probability of expecting a change at this update instance. Then we reallocate resources accommodating this new update probability distribution. As Fig. 13 shows that 
performance of our allocation policy do increase in initial runs and then it becomes steady. This is what one would expect because after a large number of runs, the update probability distribution itself becomes steady. Also we plotted 2 more graphs as shown in Fig. 14 to study the effect of delayed resource allocation. We find that resource allocation is not required to be done after every run and can be delayed without incurring any significant loss provided the monitoring phase does capture the page change behaviour nicely and also only if page change behaviour is not very erratic.

Not putting the other case when monitoring is fallacious because of no good experiment in support of it

\subsection{Performance of Scheduling algorithm}

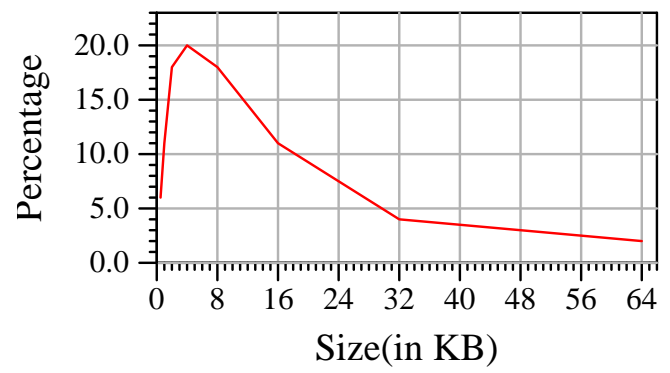

Figure 15: Size of web documents

In this experiment, we test our scheduling algorithm and show its performance. Change frequency distribution zipf parameter is set to 2 and update probability distribution zipf parameter to 1 . Sizes of the documents are generated as shown in Fig. 15 as found in [9]. Also more popular pages are set to smaller sizes in accordance with [6]. We define average probing capacity as available bandwidth divided by average size of documents. Note that this is average analysis and it is possible to make more probings than average probing capacity. As shown in Fig. 16, our scheduling algorithm performs very good and is almost lossless when number of probings is less than average probing capacity. Even when number of probings required to be scheduled exceeds average probing capacity, the loss of information incurred in scheduling phase remains quite negligible in comparison of resource allocation phase. The two kinds of losses incurred are:

1. As the number of probes to be scheduled becomes more than the average probing capacity, some probes remain undone and so some loss of information is incurred.

2. Also as number of probes becomes more and more, the chances of cases where probes scheduled for a instance exceeds the probing capacity at that instance also becomes high. So these probings get delayed for time being and hence loss of information is incurred.

\section{PROOF}

Assumption : Update distribution is uniform.

Aim : Proportional policy always performs better than Uniform policy under above assumption.

We would be comparing weighted uniform and weighted proportional policies.

Number of crawls allocated to $i^{\text {th }}$ page in proportional

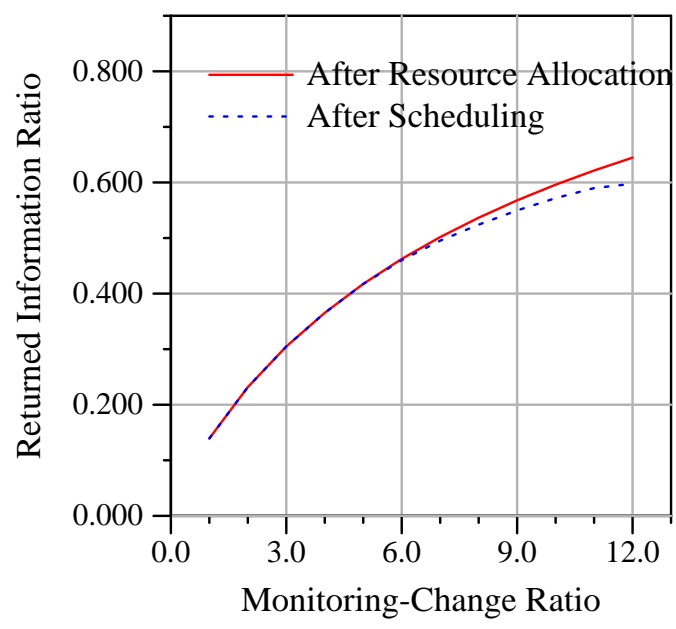

Average probing capacity $=7.6 *$ No. of Expected Changes

Figure 16: Performance Curve for different phases of scheme

policy is

$$
\frac{W_{i} * \lambda_{i}}{\sum_{i} W_{i} * \lambda_{i}}
$$

where $W_{i}$ and $\lambda_{i}$ are weight and change frequency of $i^{\text {th }}$ page respectively.

So Information gained for this page is equal to

$$
\frac{W_{i}^{2} * \lambda_{i} * \rho_{i}}{\sum_{i} W_{i} * \lambda_{i}}
$$

where $\rho_{i}$ is the update probability for $i^{t h}$ page at any update instance.

Information gained in case of Uniform Allocation for the same page is equal to

$$
\frac{W_{i}^{2} * \rho_{i}}{\sum_{i} W_{i}}
$$

So ratio of performance of Proportion to Uniform policy over all pages becomes

$$
\frac{\sum_{i} \lambda_{i} * \sum_{i} W_{i}}{\sum_{i} W_{i} * \lambda_{i}}
$$

As we know $\sum_{i} a_{i} * \sum_{i} b_{i} \geq \sum_{i} a_{i} * b_{i}$ for non-negative $a_{i}$ 's and $b_{i}$ 's, above ratio is always greater than 1 .

This proves that Proportional policy always performs better than Uniform policy no matter how page weights and change frequencies are distributed.

\section{CONCLUSIONS AND RELATED WORK}

As mentioned earlier, in our problem formulation we are not making any assumptions about the change behaviour of pages. Instead we collect and build the above statistics about a page during a monitoring period and only on the basis of this collected information, we do resource allocation. Then we keep on updating this information after every $T$ time units based on the result of the probings done. This makes our solution robust and adaptable in any web scenario. 
There have been several studies of web crawling in an attempt of capturing web dynamics. The earliest study to our knowledge is by Brewington and Cybenko. In [1], they not only studied the dynamics of web but also raise some very interesting issues for developing better crawling techniques. They showed that page change behaviour varies significantly from page to page and so crawling them equal number of times is a fallacious technique. A series of papers, [4] and [3] addresses a number of issues relating to the design of effective crawlers. In [5][16], authors approached the problem formally and devised an optimal crawling technique. A common assumption made in most of these studies is that page changes are a Poisson or memoryless process. In fact it has shown to hold true for a large set of pages but it is also found in [?] that most of web pages are modified during US working hours, i.e., 5 a.m. to 5 p.m. In our case, we go beyond these basic assumptions and present an optimal monitoring technique for answering continuous queries [12] independent of any assumption about page change behaviour. We also show that traditional crawling technique don't work well for answering of continuous queries because of the change in nature of problem as well as goal to be achieved. We formally prove that proportional allocation works better than uniform policy which differentiates the continuous and discrete query case.

There is also an altogether different approach possible for answering continuous queries where information is pushed from web pages instead of pulling it as done in our scheme [14]. Here users submit their query to the query system and then query-system registers itself to all the web pages it finds relevant to the submitted queries. Now whenever these web pages get updated, they themselves propagate their changes to the query-system which in turn report back this to the user only if it finds that changes are relevant for user query. An advantage of this approach is that here query system is no more required to monitor web pages and so there is no wastage of resources too but an obvious disadvantage of this would be dependence on web sites which may or may not propagate information at correct time, particularly when there are large number of query systems that need to be reported. Also some web sites might not even allow registration of query systems.

To our knowledge, no earlier work has focused on the aspect of probing the relevant web pages to respond to a set of continuous queries. (1) We present optimum probing techniques and also show how traditional crawling approaches won't suffice for answering continuous queries and need to be improved. We do not make any assumptions about the way in which pages change. Most of the earlier crawling strategies assume a Poisson update process. Instead of this, what we do is to record information about the nature of change of pages and keep on evolving it every time a page is probed. This makes our study more practical and robust as it is not designed with any basic assumptions. (2) Our optimization metric is defined to minimize the information loss compared to an ideal probing algorithm which probes upon every change of a page. This metric also differentiate crawling from probing. (3) We formally prove that proportional allocation policy in which the pages which are having high frequency of change are allocated more probings, works better than uniform policy which allocates equal number of probings to each page independent of its change frequency, in continuous query case which on the first sight seems to be in contradiction with a long held result that uniform is a better allocation technique than its proportional counterpart [4]. We justify this surprising behaviour and also give an intuition behind it. This shows that nature of problem of monitoring of web pages for answering continuous queries is strikingly different from the problem of devising optimal crawling techniques addressed in earlier studies.

\section{REFERENCES}

[1] B. E. Brewington and G. Cybenko. How dynamic is the Web? Computer Networks (Amsterdam, Netherlands: 1999), 33(1-6):257-276, 2000.

[2] C.Chekuri, R.Motwani, B.Natarajan, and C.Stein. Approximation techniques for average completion time scheduling. In proceedings of 8th ACM-SIAM Symposium of Discrete Algorithms, pages 609-618, 1997.

[3] J. Cho and H. Garcia-Molina. The evolution of the web and implications for an incremental crawler. In Proceedings of the Twenty-sixth International Conference on Very Large Databases, 2000.

[4] J. Cho and H. García-Molina. Synchronizing a database to improve freshness. In Proceedings of 2000 ACM International Conference on Management of Data(SIGMOD), 30(1-7):161-172, 2000.

[5] E. Coffman, J. Z. Liu, and R. R. Weber. Optimal robot scheduling for web search engines. Journal of Scheduling, 1998.

[6] C. Cunha, A. Bestavros, and M. Crovella. Characteristics of World Wide Web Client-based Traces. Technical Report BUCS-TR-1995-010, Boston University, CS Dept, Boston, MA 02215, April 1995.

[7] B. Fox. Discrete optimization via marginal analysis. Management Science, 13(3):211-216, 1966.

[8] G. N. Frederickson and D. B. Johnson. The complexity of selection and ranking in $\mathrm{x}+\mathrm{y}$ and matrices with sorted columns. Journal of Computer and System Sciences, 24:197-208, 1982.

[9] A. Heydon and M. Najork. Mercator: A scalable, extensible web crawler. World Wide Web, 2(4):219-229, 1999.

[10] T. Ibaraki and N. Katoh. Resource allocation problems: Algorithmic approaches. MIT Press, Cambridge, MA, 1988.

[11] J.K.Lenstra, A. Kan, and P.Brucker. Complexity of machine scheduling problems. Annals of Discrete Mathematics, 1:343-362, 1977.

[12] L. Liu, C. Pu, and W. Tang. Continual queries for internet scale event-driven information delivery. Knowledge and Data Engineering, 11(4):610-628, 1999.

[13] M.R.Garey, D.S.Johnson, and R.Sethi. The complexity of flowshop and jobshop scheduling. Mathematics Operation Research, 1:117-129, 1976.

[14] C. Olston, B. T. Loo, and J. Widom. Adaptive precision setting for cached approximate values. In SIGMOD Conference, 2001.

[15] J. Pitkow and P. Pirolli. Life, death, and lawfulness on the electronic frontier. In Proceedings of the Conference on Human Factors in Computing Systems CHI'97, 1997.

[16] J. Wolf, M. Squillante, P.S.Yu, J.Sethuraman, and L. Ozsen. Optimal crawling strategies for web search engines. In $W W W, 2002$.

[17] A. Wolman, G. M. Voelker, N. Sharma, N. Cardwell, A. R. Karlin, and H. M. Levy. On the scale and performance of cooperative web proxy caching. In Symposium on Operating Systems Principles, pages 16-31, 1999. 